\title{
Vitamin A Transport in Plasma of Diabetic Patients
}

\author{
Yutaka Wako, Kenichi Suzuki, ${ }^{*}$ Yoshio Goto* and \\ Shuichi Kimura \\ Laboratory of Nutrition, Faculty of Agriculture, Tohoku \\ University, Sendai 980 and *the Third Department of \\ Internal Medicine, Tohoku University School of Medicine, \\ Sendai 980
}

\begin{abstract}
Wako, Y., Suzuki, K., Goto, Y. and Kimura, S. Vitamin A Transport in Plasma of Diabetic Patients. Tohoku J. exp. Med., 1986, 149 (2), 133-143 The effects of diabetes on the vitamin A metabolism were studied. The concentrations of plasma retinol and retinyl ester were measured in diabetic patients using high-performance liquid chromatography (HPLC). In diabetic patients, the mean level of retinyl ester was significantly elevated compared to that of normal subjects (diabetes, $68.3 \pm 42.5 \mathrm{IU} / 100 \mathrm{ml}$ of plasma; study I, $87.6 \pm 64.3 \mathrm{IU} / 100 \mathrm{ml}$ of plasma; study II vs. normal, $29.8 \pm 10.3 \mathrm{IU} / 100 \mathrm{ml}$ of plasma). The ultracentrifugal and column chromatographic studies were carried out to examine the distribution of retinyl ester in patient's plasma. Ultracentrifugation study showed that the mean of $72.6 \%$ of retinyl ester was recovered in the lipoproteins of density higher than 1.006 and the remainder was recovered in the chylomicron $(d<1.006)$. Gel filtration profiles for the separation of plasma vitamin A demonstrated that retinyl ester presented in the HDL, LDL and larger molecular size lipoprotein (suspected to be VLDL) as well as in the chylomicron.

vitamin A ; diabetes; plasma lipoprotein
\end{abstract}

Vitamin A is an essential nutrient for the maintenance of various physiological functions such as growth, vision, reproduction and division of differentiation of epithelial cells (Olson 1969). A number of studies on vitamin A metabolism have been done and a great deal of knowledge has emerged concerning its mechanisms of absorption, storage and transport. Vitamin A is hydrolyzed and absorbed in the small intestine. Then retinol is reesterified and incorporated into the chylomicron and excreted into the lymph (Goodman et al. 1966). The chylomicron remnants absorbed by the liver and vitamin A are stored in the Ito's cells (Lawrence et al. 1966 ; Hirosawa and Yamada 1973). Goodman et al. (1966) showed for the first time that vitamin A circulated as retinol bound to a specific

Received August 30, 1985 ; accepted for publication April 15, 1986.

abbreviation: HDL, high density lipoprotein; LDL, low density lipoprotein ; LPL, lipoproteinlipase ; PA, prealbumin ; RBP, retinol-binding protein ; VLDL, very low density lipoprotein. 
protein, retinol-binding protein (RBP) (Kanai et al. 1968). The liver secretes the retinol-RBP complex to the circulation and the retinol is delivered to target tissues.

It has been shown that elevations of serum vitamin A levels are found in patients with chronic hypervitaminosis $\mathrm{A}$ and this elevation is mainly due to large increses in the levels of retinyl ester (Mallia et al. 1975). In the hypervitaminotic rats retinyl ester was not bound to RBP but it was found in association with the plasma lipoproteins (Smith and Goodman 1976). The effects of disease of the liver, thyroid or kidneys on this system have been evaluated (Smith and Goodman 1971). However, there are fewer studies that have looked for the level and the form of vitamin A in the plasma in diabetic patients (Murrill et al. 1941). Therefore in this study we investigated the levels of retinol and retinyl ester in patients with diabetes mellitus.

\section{Materials and Methods}

Clinical characteristics of subjects. Twelve healthy volunteers were served as the normal controls. They did not have history of diabetes and their $2 \mathrm{hr}$ postprandial blood gulcose concentrations were normal. Seventy five insulin-independent diabetic patients, who were consulted or admitted in the department of internal medicine, Tohoku university school of medicine, were studied. No patient was taking any tablet of vitamin other than drug treatment for diabetes. No volunteer also received any tablet of vitamin. The levels of plasma vitamin A, glucose and lipids were examined in forty nine patients in diabetic subjects (study I). The analyses of vitamin A levels in the plasma and the plasma fractions, which were separated by ultracentrifugation, were performed in twenty six patients in diabetic subjects (study II). The sex, age distribution and mean levels of plasma glucose, cholesterol and triglyceride in normal subjects and diabetic patients are showed in Table 1. The abnormality of glucose metabolism in these patients reported here was charaterized by the elevation of plasma glucose. There were no differences in the mean concentrations of triglyceride and cholesterol between patients (study I) and normal subjects. The total cholesterol level in 4 patients was over $250 \mathrm{mg} / 100 \mathrm{ml}$ of plasma and the triglyceride level in 7 patients was over $250 \mathrm{mg} / 100 \mathrm{ml}$ of plasma. The patients and normal subjects examined in this study had no past history of liver disease and laboratory data of liver functions such as total bilirubin, GOT, GPT, alkaline phosphatase activities were within

TABLE 1. Characteristics of the

\begin{tabular}{lcccccc}
\hline & \multicolumn{3}{c}{ Number of subjects } & & \multicolumn{2}{c}{ Age } \\
\cline { 2 - 3 } \cline { 5 - 7 } & Total & Males & Females & & Mean & Range \\
\hline Normal & 12 & 11 & 1 & & \multicolumn{2}{c}{ yrs } \\
Diabetes & & & & & & $23-38$ \\
$\quad$ Study I & 49 & 23 & 26 & & 60.8 & $29-87$ \\
$\quad$ Study II & 26 & 16 & 10 & & 59.0 & $41-76$ \\
\hline
\end{tabular}

Values are means \pm S.D.

** $p<0.01$ compared to normal subjects.

$\mathrm{NA}$, not available for analysis. 
normal ranges.

Plasma samples. Venous blood obtained after a 10-12 hr fast was drawn in heparinized tubes and the plasma was separated by centrifugation at $1,500 \times \mathrm{g}$ for $20 \mathrm{~min}$. Plasma samples were stored in the dark at $4^{\circ} \mathrm{C}$ until analysis. In study II portion of plasma was ultracentrifuged according to the method of Hatch and Lees (1968) to exclude chylomicron from the samples. $2 \mathrm{ml}$ of plasma was placed in an ultracentrifuge tube and overlayered with $1 \mathrm{ml}$ of $d=1.006$ solution. The tubes were centrifuged at $26,000 \times \mathrm{g}$ for $30 \mathrm{~min}$ at $15^{\circ} \mathrm{C}$ in a Beckman L5-65 ultracentrifuge. After centrifugation, the upper $1 \mathrm{ml}$ of the solution (containing chylomicron) was removed. The bottom fraction obteined in this way contained very low density lipoprotein (VLDL), low density lipoprotein (LDL) and high density lipoprotein (HDL), and designated as the " $d>1.006$ fraction"

High-performance liquid chromatography. HPLC was carried out with a Hitachi model 635 liquid chromatograph (Hitachi Ltd., Tokyo) that was equipped with a model 635-5002 solvent delivery system and model 650-10S fluoresence spectrophotometer. The column was $0.46 \mathrm{~cm}$ (inside diameter) $\times 25 \mathrm{~cm}$ stainless steel, packed with Nucleosil $\mathrm{C}_{18}$. The solvent was reagent grade ethanol : water, $95: 5$, filtered through a $0.5 \mu$ filter.

Standards. Purified retinyl palmitate (1,000,000 IU/g, 223-00331) was purchased from Wako Pure Chemical Industries, Ltd. Retinol was prepared from retinyl acetate (Wako Pure Chemical Industries, Ltd. 1,000,000 IU/g, 226-00321) by saponification with alcoholic $\mathrm{KOH}$ in the presence of $0.3 \%$ pyrogallol according to the J.P.IX. (1976), extracted into ethyl ether and washed with water three time. The standard stock solutions of these compounds were resolved in redistilled absolute isopropyl alcohol and the concentration was checked spectrophotometrically according to the J.P.IX. (1976). To prepare a standard curve, the standard solutions were injected into HPLC instrument and the peak heights were measured.

Analysis of plasma for retinol and retinyl ester. Plasma vitamin A was extracted according to the method described by Katui (1982). $100 \mu \mathrm{l}$ of plasma, $1 \mathrm{ml}$ of ethanol and $1 \mathrm{ml}$ of distilled water were pipeted into collared glass stopered test tube. After mixing 5 $\mathrm{ml}$ of $\mathrm{n}$-hexan was added and the contents were mixed vigorously for $5 \mathrm{~min}$. The tube was centrifuged at 1,000 rpm for $5 \mathrm{~min}$. $4 \mathrm{ml}$ of the upper layer was carefully transferred to another tube and evaporated under a nitrogen stream. For injection the residue was dissolved in $100 \mu \mathrm{l}$ of isopropyl alcohol. $25 \mu \mathrm{l}$ of this solution was injected using a micro syringe and the peak height was measured. A flow rate was $1 \mathrm{ml} / \mathrm{min}$. The concentration of retinyl ester in chylomicron was calculated by subtracting retinyl ester in the " $d>1.006$ fraction" from that in whole plasma.

Isolation of plasma lipoproteins. Lipoproteins were prepared by the method of Rudel et al. (1974). The density of the " $d>1.006$ fraction" was raised to $d=1.21$ by adding solid $\mathrm{KBr} .3 \mathrm{ml}$ of this plasma was placed in a tube and overlayered with $1 \mathrm{ml}$ of $d=1.21$ solution.

subjects and patients studied

\begin{tabular}{ccc}
\hline & Fasting plasma \\
\hline Glucose & Cholesterol & Triglyceride \\
\hline $87.9 \pm 13.3$ & $190.8 \pm 35.4$ & \\
& & $122.9 \pm 60.0$ \\
$151.6 \pm 53.3^{* *}$ & $210.7 \pm 46.0$ & $146.6 \pm 73.7$ \\
$131.7 \pm 61.9^{* *}$ & $\mathrm{NA}$ & $\mathrm{NA}$ \\
\hline
\end{tabular}


The tube was centrifuged at $114.000 \times \mathrm{g}$ for $24 \mathrm{hr}$ at $15^{\circ} \mathrm{C}$ in a Beckman L5-65 ultracentrifuge. Plasma lipoproteins (VLDL, LDL and HDL) were obtained in the upper $1 \mathrm{ml}$ of the tube $(d=1.21$ solution). This fraction was carefully removed by aspiration and dialyzed exhaustively against $0.15 \mathrm{M} \mathrm{NaCl}$ with $0.01 \%$ EDTA, $\mathrm{pH} 7.0$, at $4^{\circ} \mathrm{C}$, and designated as the " $d=1.006-1.21$ fraction".

Gel filtration of vitamin $A$ in plasma or plasma lipoproteins. $500 \mu \mathrm{l}$ of fresh plasma was applied directly to a $1.8 \times 68 \mathrm{~cm}$ Sephacryl S-200 column equilibrated with $0.05 \mathrm{M}$ phosphate buffer $\mathrm{pH} 7.4$, and $0.15 \mathrm{M} \mathrm{NaCl}$. The column was developed with the same buffer at $4^{\circ} \mathrm{C}$ in the dark. The absorbance of the eluted fraction was measured at $280 \mathrm{~nm}$ with a spectrophotometer (JASCO UVIDEC-320) and the fluorescence of vitamin A (excitation 330 $\mathrm{nm}$, emission $460 \mathrm{~nm}$ ) in the fraction was measured with a fluorescence spectrophotometer (HITACHI 204). The elution positions of retinol-binding protein (RBP), prealbumin (PA), LDL and HDL were determined by Ouchterlony immunodiffusion analyses using special antisera against RBP (20183-5), PA (20142-2), LDL (20144-6) and HDL (20105-7). These antisera were purchased from Hoechst Japan Ltd. The column chromatography of plasma lipoproteins was carried out by the method of Rudel et al. (1974). The lipoproteins,

Table 2. Plasma vitamin $A$ content in normal and diabetic subjects

\begin{tabular}{lccc}
\hline & \multicolumn{2}{c}{ Diabetes } & \multirow{2}{*}{ Normal(12) } \\
\cline { 2 - 3 } & Study I(49) & Study II(26) & \\
\hline Total V.A & $172.5 \pm 69.5$ & $230.5 \pm 91.7^{* *}$ & $173.9 \pm 25.6$ \\
Retinol & $104.2 \pm 51.2^{* *}$ & $137.1 \pm 56.4$ & $143.9 \pm 22.5$ \\
Retinyl ester & $68.3 \pm 42.5^{* *}$ & $87.4 \pm 64.3^{* *}$ & $29.8 \pm 10.3$ \\
& $39.2 \pm 15.0^{* *}$ & $35.8 \pm 15.2^{* *}$ & $17.0 \pm 5.3$ \\
\hline
\end{tabular}

V.A, vitamin A.

Values are means \pm S.D., with the number of observation in parentheses.

** $p<0.01$ compared to normal subjects.

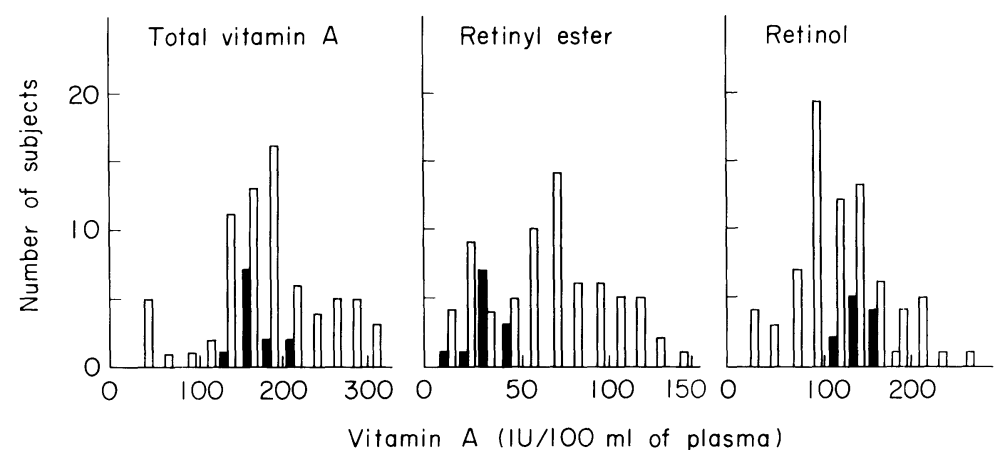

Fig. 1. Histograms of vitamin A levels in diabetic patients and normal subjects. The values are frequency of 75 diabetic patients (open boxes, study I+study II) and 12 normal subjects (solid boxes). Total vitamin A and retinol levels were classified into 13 ranks. Rank 1 is 0 to $25 \mathrm{IU} / 100 \mathrm{ml}$ of plasma and with the following ranks increasing by $25 \mathrm{IU}$. Retinyl ester levels were classified into 12 ranks. Rank $\mathrm{I}$ is 0 to $12.5 \mathrm{IU} / 100 \mathrm{ml}$ of plasma with the following ranks increasing by $12.5 \mathrm{IU}$. 



Fig. 2. HPLC chromatogram of extract of whole plasma (A), $d>1.21$ fraction (B) and $d=1.21$ fraction $(\mathrm{C})$. Peak 1, retinol; Peak 2, retinyl palmitate, Nucleosil column; eluted with mixtures of ethanol and water $95: 5$. Detection, fluorescence (Ex. $330 \mathrm{~nm}, \mathrm{Em} .460 \mathrm{~nm}$ ).

which was prepared by the ultracentrifugation (" $d=1.006-1.21$ fraction"), was applied to an agarose column, $1.5 \times 100 \mathrm{~cm}$ Bio-Gel A-5 m 200-400 mesh, equilibrated with $0.15 \mathrm{M}$ $\mathrm{NaCl}, 0.01 \%$ EDTA, $\mathrm{pH} 7.0$. The column was eluted with the same solution at a flow rate of $10 \mathrm{ml} / \mathrm{hr}$. The other Bio-Gel A chromatography procedure was similar to that of the Sephacryl S-200 chromatography described above.

Analysis of glucose and lipids in plasma. Plasma'glucose, triglyceride and cholesterol levels were determined by the methods of Miwa et al. (1972), Nishina (1978) and Allain et al. (1974) respectively.

Statistical analysis. Values are given as means \pm S.D. Differences between mean values were analyzed with Student's $t$-test and judged to be significant when $p$ values were less than 0.05 .

\section{RESULTS}

Plasma vitamin A levels in diabetic patients

The mean concentrations of total plasma vitamin A, retinol, retinyl ester and

TABLE 3. Distribution of vitamin $A$ in fractions obtained after ultracentrifugation of plasma in diabetic patients (study II)

\begin{tabular}{lc}
\hline & IU $/ 100 \mathrm{ml}$ of plasma \\
\hline Retinyl ester in $d<1.006$ fraction & $27.2 \pm 50.0$ \\
Retinyl ester in $d>1.006$ fraction & $60.2 \pm 48.4$ \\
& $\%$ \\
$\%$ of retinyl ester in $d>1.006$ fraction & $72.6 \pm 26.8$ \\
\hline
\end{tabular}

Values are means \pm s.D. in 26 diabetic patients. 


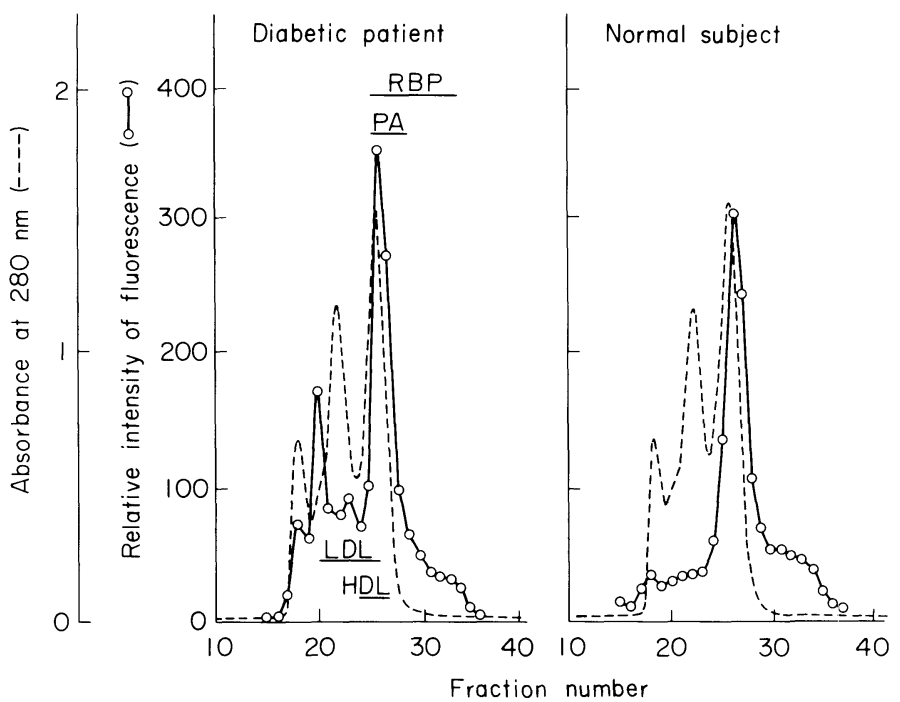

Fig. 3. Gel filtration profile of vitamin A in human plasma. $500 \mu \mathrm{l}$ of fresh plasma from diabetic patient and normal subject were chromatographed on Sephacryl S-200. The effuluents were assayed for protein ( ....... absorbance at $280 \mathrm{~nm}$ ) and vitamin A ( ○—— , fluorescence at Ex. $330 \mathrm{~nm}$, Em. $460 \mathrm{~nm})$. The elution position of LDL, HDL, RBP and PA (horizontal bars) were determined by Ouchterlony immunodiffusion assay.

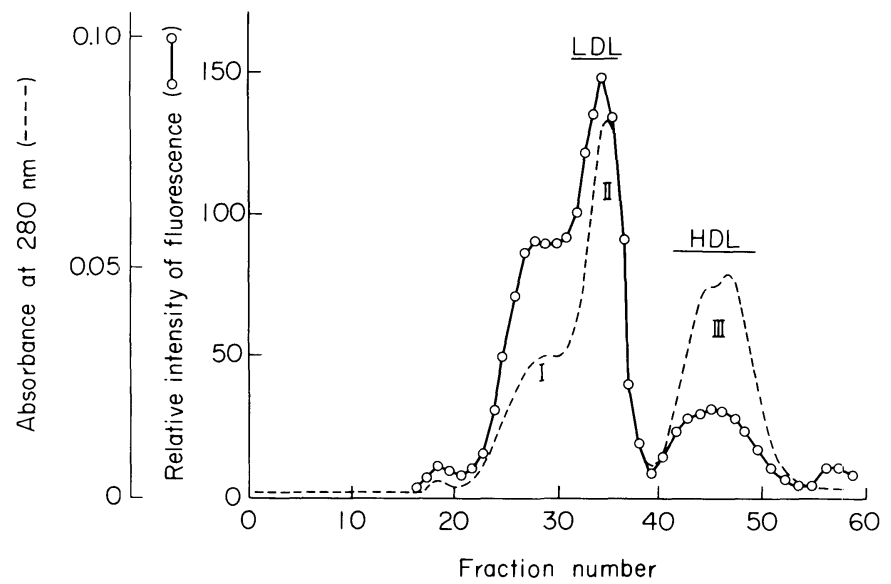

Fig. 4. Demonstration of the presence of vitamin $\mathrm{A}$ in plasma lipoprotein. Lipoprotein from diabetic patient was chromatographed on Bio-Gel A-5 m. The eluted fractions were assayed for absorbance at $280 \mathrm{~nm}(\ldots \ldots)$ and for fluorescence at Ex. $330 \mathrm{~nm}, \mathrm{Em} .460 \mathrm{~nm}(\mathrm{O}-\mathrm{O}$, as a measure of vitamin A). The elution positions of LDL and HDL (horizontal bars) were determined by Ouchterlony immunodiffusion assay. 
the proportion of vitamin A presented as retinyl ester in normal subjects and

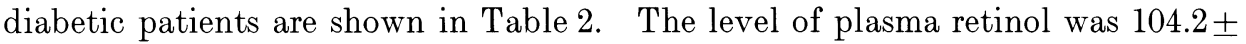
51.2 (study I), $137.1 \pm 56.4$ (study II) IU/100 ml of plasma for patients and $143.9 \pm$ $22.5 \mathrm{IU} / 100 \mathrm{ml}$ of plasma for normal subjects. Retinyl ester levels were $68.3 \pm$ 42.5 (study I), $87.4 \pm 64.3$ (study II) IU/100 ml of plasma for patients and $29.8 \pm$ $10.3 \mathrm{IU} / 100 \mathrm{ml}$ of plasma for normal subjects. Thus in patient group the mean levels of retinyl ester and the proportion of vitamin A presented as retinyl ester increased markedly compared to levels and proportion in normal subjects. In 16 patients more than $50 \%$ of vitamin A was present as retinyl ester. Histograms of vitamin A levels in diabetic patients and normal subjects are shown in Fig. 1. While in normal subjects all retinyl ester levels were less than $50 \mathrm{IU} / 100 \mathrm{ml}$ of plasma, in diabetic patients more than $50 \mathrm{IU} / 100 \mathrm{ml}$ of retinyl ester levels were frequently found.

\section{Distribution of retinyl ester in diabetic patient's plasma}

An ultracentrifugation study was carried out to examine the distribution of retinyl ester in plasma (Fig. 2). HPLC chromatogram of whole plasma extract showed the presence of plasma retinol and retinyl ester (Fig. 2A). After the ultracentrifugation of plasma at $d=1.21$, HPLC monitering demonstrated that retinyl ester was recovered in the upper fraction (which contained lipoproteins) and retinol was present in the bottom fraction (Fig. 2B, C). These results demonstrated that all of the retinyl ester existed in the lipoproteins. To investigate the level of retinyl ester presented in the chylomicron and lipoproteins of density higher than 1.006 in patients plasma (study II), the ultracentrifugation study was performed at $d=1.006$ (Table 3 ). The mean levels of retinyl ester in the chylomicron and the other lipoproteins (" $d>1.006$ fraction") were $27.2 \mathrm{IU} / 100$ $\mathrm{ml}$ of plasma and $60.2 \mathrm{IU} / 100 \mathrm{ml}$ of plasma. Therefore a mean of $72.6 \%$ of total retinyl ester was present in the lipoproteins of density higher than 1.006 in the patient's plasma.

\section{Gel filtration profiles for the separation of plasma vitamin $A$}

To observe the presence of retinyl ester in plasma lipoproteins, gel filtration analyses were also carried out (Figs. 3,4). Plasmas from patients and normal subjects were chromatographed on a Sephacryl S-200 column. A typical gel filtration profile showed that vitamin A was eluted in a volume which was characteristic of RBP-PA complex (fraction 25-28) in both plasmas. In the plasma of patients vitamin A was also found in a volume which was characteristic of the plasma lipoproteins (fraction 19-24). In order to investigate the precise distribution of retinyl ester among the lipoprotein classes, plasma lipoproteins (" $d=1.006-1.21$ fraction" which does not contain chylomicron) were chromatographed on a column of Bio-Gel A-5 m. The typical elution patterns for the separation of lipoproteins are shown in Fig. 4. The lipoproteins was separated 
into three peaks. Peak II and peak III fractions cross reacted with anti-LDL and anti-HDL respectively. Therefore it was shown that peak II and peak III fractions contained LDL and HDL. The peak I fraction would be expected to contain VLDL according to its molecular size and Rudel's observation. These gel filtration experiments directly demonstrated the presence of retinyl ester in the HDL, LDL and larger molecular size lipoproteins.

\section{Discussion}

Vitamin A is normally mobilized from the liver (storage site for vitamin A) as the unesterified free alcohol, retinol, which is bound to its specific carrier protein, RBP (Kanai et al. 1968) and is delivered to target tissues. Therefore in fasting plasma, almost all of the vitamin $\mathrm{A}$ is in the form of retinol bound to the $\mathrm{RBP}$ and a smaller part is in the form of retinyl ester (Kanai et al. 1968 ; Smith and Goodman 1976). The present studies were undertaken to examine the effects of diabetes on the form and distribution of vitamin $\mathrm{A}$ in plasma. In patients with diabetes, the level of retinyl ester increased markedly in plasma obtained after a 10-12 hr fast (Table 2, Fig. 1). Until now the presence of large amount of retinyl ester in fasting plasma has not been reported except in patients with hypervitaminosis A. Smith and Goodman (1976) suggested that in patients with chronic hypervitaminosis A, the large elevations in total vitamin A were observed accompanied with the elevation of retinyl ester levels. However, in the patients with diabetes reported here, extreme elevation could not be observed in total vitamin A or retinol levels (Table 1, Fig. 1). Then to determine the distribution of this abnormal retinyl ester, an ultracentrifugation study was carried out (Table 3, Fig. 2). Fig. 2 shows that all of the retinyl ester was found in association with the lipoprotein fractions. This result confirmed Mallia et al.'s (1975) observation in hypervitaminotic rats, virtually all of the retinyl ester was found in the lipoproteins of density less than 1.21 (Smith and Goodman 1971). Moreover to examine the amount of retinyl ester presented in the chylomicron, next ultracentrifugation study was performed. Table 3 shows only a mean of $27.4 \%$ of the retinyl ester is recovered in the chylomicron $(d<1.006)$. Accordingly these results presented here suggest that a considerable level of retinyl ester is frequently found in patients with diabetes mellitus without extreme elevation of total vitamin A. Almost all of this retinyl ester was in association with the lipoproteins of density higher than 1.006 and the remainder of the retinyl ester was in association with chylomicron.

There findings have been confirmed by the chromatograph investigation of the plasma and plasma lipoproteins. The typical gel filtration profiles for the separation of plasma vitamin A demonstrated that the greater part of the vitamin A was found in a volume which was characteristic of the RBP-PA complex, while in diabetic patients a considerable amount of vitamin $\mathrm{A}$ was also found in a volume which was characteristic of the plasma lipoproteins (Fig. 3). This obser- 
vation supported the hypothesis that retinyl ester was present in the lipoproteins. Since a mean of $72.6 \%$ of the retinyl ester was recovered in the lipoproteins of density higher than 1.006, it was necessary to ascertain how these retinyl esters were distributed among the lipoprotein classes. The separation of the lipoprotein classes was performed by an agarose column chromatography (Fig. 4). The results of the gel filtration clearly demonstrated that retinyl ester was distributed to the HDL, LDL and larger molecular size lipoproteins (suspected to be VLDL). Accordingly, the ultracentrifugal and column chromatographic studies presented here suggest that these lipoproteins participate in the transport of vitamin A in plasma in the diabetic patients.

The mechanism responsible for the appearance of retinyl ester in the lipoproteins in fasting plasma is not entirely clear. In hypervitaminosis A, the liver is filled with vitamin A and excess vitamin A overflows to the circulation. It has been established that the RBP molecule contains one binding site for one molecule of retinol (Kanai et al. 1968; Muto et al. 1972). Thus Mallia et al. (1975) suggested that the large increases in the levels of total vitamin A, greatly exceeding levels of serum RBP, caused the appearance of some of the retinol and all of the retinyl ester in the serum lipoproteins. While in patients with diabetes, extreme elevation of total vitamin A was not observed. Therefore a different mechanism should be responsible for the appearance of vitamin A in the lipoproteins of diabetic patients. An abnormal lipoprotein metabolism has been demonstrated in patients with diabetes (Carlson and Bottiger 1972). Triglyceride is removed from chylomicron at peripheral tissue by the action of lipoprotein lipase (LPL), and ultimately chylomicron turns into chylomicron remnant (Brown et al. 1981). Taskinen et al. (1982) reported that in insulin-independent diabetic patients, LPL activity was decreased in adipose tissue. Accordingly, in diabetic patients it seems that triglyceride romoval efficiency decreases and clearance of triglyceride-rich particles such as, chylomicron, impaires. If these changes occur it may be possible that the retinyl ester is easy to transconvert from chylomicron to another lipoprotein before absorbed by the liver and retinyl ester accumulate in plasma in diabetic patients. However, this speculation is inconsistent with the fact that the elevation of retinyl ester was also found in patients who had normal triglyceride level in our data. Anyway some abnormal changes in lipoprotein metabolism must be contributed to the elevation of retinyl ester. The age of the patient group was much higher than that of the normal subject group (Table 1). The age may also be related to the elevation of retinyl ester presented in the lipoproteins. To elucidate this mechanism further study will be needed.

It has been shown that excessive intake of vitamin A leads to a distinct hypervitaminotic, toxic manifestation and this toxic effects of vitamin A seem to be caused by the activation of proteolytic enzymes (Bangham et al. 1964 ; Roels et al. 1969 ; Clark 1971). However, these toxic effects of vitamin A were not seen 
when retinol was a form bound to the RBP (Dingle et al. 1972). It was also suggested that serum lipoproteins might nonspecifically deliver vitamin A to peripheral tissue. Thus it seems that increased plasma retinyl ester (which is not bound to RBP but bound to lipoprotein) levels might be related to vitamin A intoxication. Thus in diabetic patients who have high level of retinyl ester even normal level of total vitamin A, intoxication such as epithelial abnormalities or impairment of biological membranes, may occur.

\section{Acknowledgments}

We are greateful to Dr. Kiyoshi Oba for discussion and to Dr. Kyosuke Masaki for helpful advice on the analysis of vitamin A by HPLC.

\section{References}

1) Allain, C.C., Poon, L.S., Chan, C.S., Richmond, W. \& Fu, P.C. (1974) Enzymatic determination of total cholesterol. Clin. Chem., 20, 470-475.

2) Bangham, A.D., Dingle, J.T. \& Lucy, J.A. (1964) Studies on the mode of action of excess of vitamin A. IX. Penetration of lipid monolayer by compounds in the vitamin A series. Biochem. $J$., 90, 133-140.

3) Brown, M.S., Kovanen, P.T. \& Goldstein, J.L. (1981) Regulation of plasma cholesterol by lipoprotein receptors. Science, 212, 628-635.

4) Carlson, L.A. \& Bottiger, L.E. (1972) Ischemic heart-disease in relation to fasting values of plasma trigrycerides and cholesterol. Lancet, 1, 865-871.

5) Clark, L. (1971) Hypervitaminosis A : a review. Aust. vet. J., 47, 568-571.

6) Dingle, J.T., Fell, H.B. \& Goodman, DeW.S. (1972) The effect of retinol and retinolbinding protein on embryonic skeletal tissue in organ culture. J. Cell Sci., 11, 393402.

7) Goodman, Dew.S., Blomstrand, R., Werner, B. \& Shiratori, T. (1966) The intestinal absorption and metabolism of vitamin A and B-carotene in man. J. clin. Invest., 45, $1615-1623$.

8) Hatch, F.T. \& Lees, R.S. (1968) Advances in Lipid Research, Academic press, New York, Vol. 6, pp. 1-68.

9) Hirosawa, K. \& Yamada, H. (1973) The localization of the vitamin A in the mouse liver as revealed by electron microscope radioautography. J. Elect. Microsc., 22, 337346.

10) Kanai, M., Raz, A. \& Goodman, DeW.S. (1968) Retinol binding protein : The transport protein for vitamin A in human plasma. J. clin. Invest., 47, 2025-2044.

11) Katui, G. (1982) Assay methods of vitamin A. Vitamins, 56, 97-101. (Japanese)

12) Lawrence, C.W., Crain, F.D., Lotspeich, F.J. \& Krause, R.F. (1966) Absorption, transport, and storage of retinyl- $15-{ }^{-14} \mathrm{C}-$ palmitate- $9,10-{ }^{3} \mathrm{H}$ in the rat. J. Lipid Res., 7, 226-229.

13) Mallia, A.K., Smith, J.E. \& Goodman, DeW.S. (1975) Metabolism of retinol-binding protein and vitamin A during hypervitaminosis A in the rat. J. Lipid Res., 16, 180188.

14) Miwa, I., Okuda, J., Maeda, K. \& Okuda, G. (1972) Mutarotase effect colorimetric determination of blood glucose with B-D-glucose oxidase. Clin. chim. Acta, 37, 538543.

15) Murrill, W.A., Horton, P.B., Leiberman, E. \& Newbrurgh, L.H. (1941) Vitamin A and carotene. II. Vitamin A and carotene metabolism in diabetics and normals. $J$. clin. Invest., 20, 395-400. 
16) Muto, Y., Smith, J.E., Milch, P.O. \& Goodman, DeW.S. (1972) Regulation of retinolbinding protein metabolism by vitamin A status in the rat. J. biol. Chem., 247, $2542-2550$.

17) Nishina H. (1978) Neutral lipid. J. med. Tech., 22, 1304-1313. (Japanese)

18) Olson, J.A. (1969) Metabolism and function of vitamin A. Fed. Proc., 28, 16701677 .

19) Roels, O.A., Anderson, O.R., Lui, N.S.T., Shah, D.O. \& Trout, M.E. (1969) Vitamin A and membranes. Amer. J. clin. Nutr., 22, 1020-1032.

20) Rudel, L.L., Lee, J.A., Morris, M.D. \& Felts, J.M. (1974) Characterization of plasma lipoproteins separated and purification by agarose column chromatography. Biochem. J., 139, 89-95.

21) Smith, F.R. \& Goodman, DeW.S. (1971) The effects of disease of the liver, thyroid, and kidneys on the transport of vitamin A in human plasma. J. clin. Invest., 50, 2426-2436.

22) Smith, F.R. \& Goodman, DeW.S. (1976) Vitamin A transport in human vitamin A toxicity. New Engl. J. Med., 294, 805-808.

23) Taskinen, M.R., Nikkila, E.A., Kuusi, T. \& Haruno, K. (1982) Lipoprotein lipase activity and serum lipoproteins in untreated type 2 (insulin independent) diabetes associated with obesity. Diabetologia, 22, 46-55.

24) The Japanese Pharmacopeia IX. 212-214 (1976) 